\title{
Rasgos distintivos, binarismo y definición de fonemas: sobre la influencia de Jakobson en Emilio Alarcos Llorach
}

\author{
Phonemes' distinctive features, binarism and definition: \\ on Jakobson's influence on Emilio Alarcos Llorach \\ Francisco Javier Perea Siller \\ Universidad de Córdoba (España). Correo electrónico: felpesif@uco.es
}

\begin{abstract}
El objetivo de este artículo es analizar el grado de influencia que ejercieron algunas ideas principales de Roman Jakobson en la Fonología española de Emilio Alarcos Llorach, a lo largo de las cuatro ediciones que conoció $(1950,1954,1961$ y 1965). El lingüista español intenta conciliar las orientaciones fonológicas de Trubetzkoy y Martinet con la que ofrece Jakobson, quien desde 1939 apostó por el binarismo en la explicación de los rasgos distintivos y la definición de los fonemas. Merece la pena señalar que en algunos pasajes Alarcos sigue de cerca los presupuestos jakobsonianos en la primera y segunda ediciones, mientras que se aleja de la explicación binarista en la tercera y cuarta. Por el contrario, lugares en los que Alarcos sigue a Trubetzkoy y Martinet permanecen inalterados durante los años que median entre 1950 y 1965.
\end{abstract}

Palabras claves: rasgo distintivo, oposición fonológica, fonema, binarismo, Fonología.

The aim of this article is to analyze the degree of influence of some relevant ideas by Roman Jakobson on Emilio Alarcos Llorach's Fonología Española, along the four editions that the book had (1950, 1954, 1961 y 1965). The Spanish linguist tries to harmonize the phonological guidelines of Trubetzkoy and Martinet, and the one of Jakobson. In effect, from 1939 Jakobson made a determined commitment to binarism in the explanation of distinctive features and definition of phonemes. It is worth noting that Alarcos pays close attention to Jakobsonian theoretical assumptions in the first and second editions, but he departs from binarist explanation in the third and fourth ones. On the contrary, passages in which Alarcos follows Trubetzkoy and Martinet remain unchanged over the years between 1950 and 1965.

Keywords: distinctive feature, phonological oppositions, phoneme, binarism, Phonology. 
La aparición en 1950 de la Fonología española de Emilio Alarcos Llorach supuso una de las primeras aplicaciones a una lengua particular de la fonología nacida en el Círculo Lingüístico de Praga ${ }^{1}$. Se registraba un influjo directo de los Grundzüge der Phonologie (1939) de Trubetzkoy, como es bien conocido. Pero además de la obra póstuma del lingüista ruso, otras influencias decisivas fueron las de Jakobson y Martinet, tanto en la fonología sincrónica como en la diacrónica ${ }^{2}$. El objetivo de este artículo es mostrar la huella decisiva que imprime Roman Jakobson en la Fonología española, en todo lo referente al análisis de los fonemas en rasgos distintivos y en la adopción de un modelo binarista.

Adoptamos una perspectiva que abarca la evolución que ha experimentado la obra de Alarcos desde la primera edición, en 1950 hasta la cuarta, en 1965. El análisis permitirá comprobar que en 1950, en el análisis de los fonemas según los rasgos distintivos, la propuesta teórica se acerca más a Jakobson que a Trubetzkoy, tanto en la parte general como en la clasificación de los fonemas consonánticos del español. En la segunda edición (1954), el lingüista español seguirá la evolución de Jakobson en el desarrollo de la exposición binarista de los rasgos distintivos; pero en 1961, fecha de la tercera edición, se registra un cierto desapego de la teoría del maestro ruso afincado en los Estados Unidos, que repite en la cuarta edición y última, de 1965.

\section{LA DEFINICIÓN DEL FONEMA}

El concepto de fonema que divulga Trubetzkoy en los Principios de Fonología se debe expresamente a Jakobson. De él toma las definiciones que aparecen en el cuerpo del texto. La primera de ellas se expresa como sigue: "Las unidades fonológicas que, desde el punto de vista de la lengua tratada, no pueden ser analizadas en unidades fonológicas aún más pequeñas y sucesivas, serán llamadas fonemas" (Trubetzkoy 1973 [1939]: 32). La definición adapta esta otra de Jakobson, defendida en 1929 en su trabajo "Remarques sur l'evolution phonologique du russe": "Tous termes d'opposition phonologique non susceptibles d'être dissociés en sous-oppositiones plus menues sont appelés phonèmes" (1971 [1929]: 8). Nótese que Trubetzkoy ha añadido la palabra sucesivas. Se trata de un cambio que se introdujo en el "Projet de terminologie phonologique standarisée" (1931:311).

La importancia otorgada a los rasgos distintivos llevará a Jakobson a replantear la definición misma de fonema que había dado en 1929. Tanto Trubetzkoy (1973 [1939]: 34) como el propio Jakobson en una conferencia de 1962 evocan el año 1932 como el momento en que el segundo ofrece su nueva propuesta de definición de fonema: "By this term we designate a set of those concurrent sound properties which are used in a given language to distinguish words of unlike meaning" (1971 [1932]: 231). En la misma conferencia, Jakobson (1971 [1962]: 636) señala que

Precedían a este libro dos artículos, aunque uno de ellos se publicaría un año después de la Fonología española (Alarcos 1949, 1951).

En un análisis de las cuatro ediciones de la Fonología española, Perea (2014) trata la creciente influencia del distribucionalismo norteamericano en los capítulos de fonología general y sincrónica, y la de Martinet en la fonología diacrónica. 
después adoptó la expresión rasgos distintivos (distinctive features), tomándolos de Bloomfield y Sapir en $1933^{3}$.

Trubetzkoy también recoge esta segunda definición del fonema: "el fonema es el conjunto de las particularidades fonológicamente pertinentes de una imagen fónica" (1973 [1939]: 34). Hay que señalar que, aunque Trubetzkoy se refiere a estas particularidades pertinentes de los fonemas, la suya es ante todo "una teoría de sistemas de fonemas: constructos 'segmentales' ideales reducidos a su mínimo de distintividad e identificados sólo por su oposición a otros elementos del mismo sistema" (Anderson 1990: 141). Ello significa que la idea de los fonemas como compuestos de rasgos "se debe, en realidad, a Jakobson y es, en cierto modo, extraña al sentido real de la obra de Trubetzkoy" (1990: 141)

Como se sabe, en los años posteriores, Jakobson llevará los rasgos distintivos al centro del sistema fonológico. Así, escribirá en un texto de 1949:

El fonema no es por sí mismo el término de oposición. Por ejemplo el fonema $b$ no exige de modo irrevocable, irreversible y necesario ninguna oposición definida, mientras que sí lo exige cualquier rasgo distintivo. La tensión no es distintiva más que frente al relajamiento, la gravedad frente a la acuidad y la saturación alta frente a la baja, y, tal como decía Saussure, la presencia de la resonancia nasal no es distintiva más que en oposición a su ausencia y viceversa. El principio dicotómico se aplica aquí de modo absoluto. (1984 [1949]: 188).

Alarcos será receptivo a la teoría del fonema que desarrolla Jakobson más allá de las definiciones que aparecen en los Principios de Trubetzkoy. Se puede afirmar que partiendo del marco general ofrecido por este, desde la primera edición Alarcos otorga gran importancia a los rasgos distintivos, como se puede comprobar desde las mismas definiciones que ofrece de fonema. En el primer capítulo ("Fonología de la palabra: función distintiva") introduce los conceptos de oposición, los rasgos pertinentes, la diferencia entre fonemas y sonidos y el método para descubrir tales diferencias, el sistema fonológico, la clasificación de las oposiciones fonológicas y los conceptos de neutralización y archifonema. En este capítulo aparece la definición de los Principios: "A estas unidades fonológicas que, en una lengua dada, no son divisibles en unidades sucesivas más pequeñas o simples, se les da el nombre de fonemas (1950: 24). Las cursivas son del autor, y preparan para la definición que aparecerá en seguida, originariamente de Jakobson y presente en Trubetzkoy: "Puede definirse el fonema como el conjunto de la propiedades relevantes de un complejo fónico".

Hasta aquí, se reproduce de la teoría expuesta en los Principios, pero ya desde 1950 Alarcos va más allá, como lo muestra el epígrafe 19. Merece la pena la transcripción:

Quizá estas referencias se puedan interpretar como una manera de señalar la precedencia de la idea sobre sus colegas norteamericanos. En todo caso, aunque el término aparezca en Bloomfield, a juicio de Anderson (1990: 296), el lingüista norteamericano no le otorgó ni mucho menos la importancia que le concedería Jakobson y que continuaría creciendo en los años posteriores.

\footnotetext{
Martinet escribirá en el mismo sentido: "Troubetzkoy est mort avant d'avoir pu formuler une théorie des traits distinctifs que était latente dans son oeuvre" (1965: 127). Son interesantes los comentarios de Akamatsu (1988: cap. 3) sobre las diferencias existentes en la teoría de los rasgos distintivos en Trubetzkoy y Jakobson.
} 
Pero, entonces, puede preguntarse: ¿cuál es la unidad fónica más simple: el fonema o cada uno de estos caracteres válidos fonológicamente? Vemos que así como las palabras se distinguen gracias a los fonemas, éstos se distinguen y oponen entre sí gracias a sus rasgos pertinentes o diferenciales. Llamamos, pues, rasgo pertinente, relevante, válido o distintivo, a toda característica fónica susceptible de diferenciar por sí sola el sentido intelectual de una palabra [...]. Son los rasgos pertinentes los que aseguran, por tanto, la función diferencial, y deben ser las unidades básicas de la fonología. (1950: 25).

Este pasaje no debe pasar desapercibido, porque en nuestra opinión presenta un contenido revolucionario. A pesar de que el rasgo distintivo se toma en otros autores como un paso necesario en la descripción fonológica ${ }^{5}$, la teoría que Alarcos esboza en la primera edición de la Fonología española enlaza con la teoría de Jakobson que desarrolla a partir de 1938, una teoría que poco a poco desplazará el interés de los fonemas hacia los rasgos distintivos, y que llevará en el generativismo a prescindir prácticamente del concepto de fonema ${ }^{6}$.

\section{LA HIPÓTESIS BINARISTA DE $\mathrm{JAKOBSON}^{7}$}

La hipótesis binarista sobre las oposiciones fonológicas no fue desarrollada por Jakobson hasta la muerte de Trubetzkoy, aunque anteriormente ya habían discrepado acerca de la conveniencia de reconocer las oposiciones multilaterales, frente a las bilaterales (Anderson 1990: 142). Trubetzkoy había escrito:

En las oposiciones bilaterales la base de comparación, es decir, el conjunto de particularidades que los dos miembros de la oposición poseen en común es propia sólo de éstos, es decir, que no aparece en ningún otro miembro del mismo sistema. En cambio, la base de comparación de una oposición multilateral no es exclusiva de los dos miembros de esa oposición, sino que aparece también en otros miembros del mismo sistema. (1973 [1939]: 60).

En cambio, en opinión de Jakobson, las oposiciones multilaterales pueden reducirse a combinaciones de oposiciones bilaterales. La hipótesis se desarrolló en una comunicación presentada en 1938 y publicada en 1939: "Observations sur le classements phonologiques des consonnes”, así como en los primeros trabajos sobre el lenguaje infantil y la afasia, presentados o publicados entre 1939 y $1941^{8}$. Jakobson

Si bien, como veremos, Martinet no acepta el devenir binarista de la teoría de los rasgos pertinentes que planteará Jakobson, también recoge en su obra la importancia del rasgo distintivo en el análisis fonológico, hasta el punto de afirmar: "Ce n'est pas le phonème, mais le trait pertinent qui est l'unité de base de la phonologie" (1949: 46).

6 Así se entiende un aserto como el de la reciente Fonética y fonología de la RAE, en la que se lee: "Las unidades básicas de la fonología clásica eran los fonemas; la fonología actual, en cambio, se basa en el concepto de rasgo distintivo" (2011: 2.8a).

7 Además de las propias obras de Jakobson citadas en la bibliografía, pueden consultarse sobre este aspecto los trabajos de Lepschy (1971), Alcina y Blecua (1975: 2.2.5.6.0, n. 11), Gil (1989) y Anderson (1990: cap. 5).

$8 \quad$ El origen de estos trabajos fue su comunicación al V Congreso Internacional de Lingüistas (Bruselas, 1939), que se publicaría diez años más tarde como "Les lois phoniques du langage enfantin et leur place dans la phonologie 
plantea la adquisición lingüística como un proceso de distinciones progresivas de tipo binario. Hay que señalar que estos textos aparecen citados desde la primera edición de la Fonología española (1950) de Alarcos.

En el artículo de 1939, Jakobson afirma, por ejemplo, que "todo sistema vocálico en general obedece al principio de la dicotomía y se deja reducir a un número restringido de cualidades fonológicas que forman oposiciones binarias" (1976 [1939]: 132). Lo mismo ocurre con las consonantes: a pesar de que los puntos de articulación son cuatro (velares, palatales, dentales, labiales), pueden reducirse a dos oposiciones: graves y agudas y posteriores / anteriores ${ }^{9}$.

En Lenguaje infantil, afasia y leyes generales de la estructura fónica (1941), Jakobson relaciona la constitución binaria de las oposiciones con el orden de adquisición del lenguaje por el niño, y con la estructura universal de las lenguas ${ }^{10}$.

En Estados Unidos se desarrolla una segunda fase de la teoría del binarismo fonológico. Los principales escritos datan de finales de la década de 1940 y principios de la de 1950: "On the Identification of the Phonemic Entities" (1949) y "For the Correct Presentation of Phonemic Problems" (1951), ambos citados por Alarcos en la segunda edición de su Fonología ${ }^{11}$. En el primero de estos artículos ya aparece el análisis de los fonemas en sus rasgos distintivos en servo-croata. La forma de presentación de los rasgos es de tipo binario, marcándose con un signo + si se da el primer término de la oposición y con el signo - si se da el segundo.

Este análisis obtendrá su culminación en el libro en colaboración con Fant y Halle Preliminaries to Speech Analysis (1952). En esta obra se intenta describir los doce rasgos distintivos presentes en las lenguas del mundo. Algunos de estos rasgos toman una terminología diferente de las obras anteriores, que también será recogida por Alarcos desde la segunda edición de la Fonología española.

Posteriormente, Jakobson seguirá depurando la hipótesis binarista en otras publicaciones, también recogidas por Alarcos en sus ediciones tercera y cuarta de su obra $^{12}$, aunque ya la influencia se ha detenido, pues como veremos Alarcos se separa del binarismo.

générale", inserto en la traducción de Cantineau de los Principios de Trubetzkoy (París, 1949). En relación con este documento también está el extenso artículo "Kindersprache, Aphasie und Allgemeine Lautgesetze", escrito entre Oslo y Estocolmo entre 1939 y 1941, y publicado en forma de libro en Upsala, 1941. La traducción española de 1974 contiene otros escritos posteriores acerca de la adquisición lingüística y la afasia.

9 Respecto del concepto de oposición binaria, se ha señalado que Jakobson se basa en la lógica de Husserl (Gil 1989) para establecer dos tipos de oposiciones: la de términos contradictorios y la de términos contrarios: la primera viene definida por la presencia o ausencia de un mismo elemento, y la segunda por la polaridad entre un máximo y un mínimo de una misma cualidad (1976 [1939]: 132).

10 Escribe Jakobson en las conclusiones: "vemos [...] que las adquisiciones fónicas del niño y los trastornos fónicos del afásico se basan en las misas leyes de solidaridad que el inventario fónico y la historia de los sonidos de todas las lenguas del mundo" (1974 [1941]: 135). Hay que señalar que en publicaciones posteriores, matiza la afirmación de la relación especular entre la adquisición y la pérdida del sistema fónico.

11 Entre otros lugares, 1954: 38 y 69, y 1954: 69, respectivamente.

12. Especial repercusión obtendrá Jakobson y Halle (1956). También Halle había defendido el binarismo en esos años, principalmente en 1954 y en 1957, de los que Alarcos ha citado el primero. 


\section{Alarcos y la hipótesis BinARISta}

\subsection{El lugar de articulación}

A lo largo del capítulo "Función distintiva: los rasgos pertinentes" (§§36-50), se tratan los rasgos pertinentes de las consonantes. Un lugar donde se muestra la influencia de los planteamientos binaristas de Jakobson, como desarrollo de los postulados de Trubetzkoy, es en el epígrafe 41 y siguientes, en que se trata la localización de las consonantes. El problema se sitúa en torno a las propiedades distintivas que se basan en la diferente localización de los sonidos: "Sin duda, la localización de los fonemas consonánticos es un rasgo diferencial, pero no opone los fonemas bilateralmente, sino multilateralmente: se distinguen varias series de localización en las que se agrupan los fonemas realizados en un mismo punto de articulación" (1950: §41).

El problema fonológico que se suscita es cómo conseguir una descripción binaria en un sistema de oposiciones multilaterales. En las dos primeras ediciones, el epígrafe 41 se cerraba con la siguiente interrogación: “¿No habrá otras relaciones entre los fonemas de las diversas localizaciones, de manera que unos a otros se opongan bilateralmente?".

La respuesta a la cuestión es el objeto del epígrafe 42, central, en nuestra opinión, en cuanto a las ideas de Alarcos en estos momentos. Sigue un artículo de Jakobson, "Observations sur le classement..." (1939), en el que las diferencias de localización se reducen a rasgos binarios de tipo acústico. En el sistema de Alarcos, las consonantes que por la localización se distinguen en cuatro grupos, desde el punto de vista acústico se agrupan en dos oposiciones bilaterales (1950: §42):

- Consonantes labiales y velares presentan timbre grave (por el efecto de un resonador único).

- Consonantes palatales y dentales presentan timbre agudo (resonador dividido).

- Consonantes velares y palatales se articulan en la parte posterior.

- Consonantes dentales y labiales se articulan en la parte anterior.

En la segunda edición, Alarcos sigue ahora los Preliminaries de Jakobson, Fant y Halle (1952), en que se profundiza en las características acústicas de estas oposiciones, de manera que la oposición anterior / posterior, es sustituida por difuso / denso:

Desde el punto de vista acústico, el espectrograma [...] muestra para las velares y palatales una relativa densidad de los formantes de frecuencia en la zona central, mientras para las dentales y labiales muestra una relativa difusión o dispersión de estos formantes. Por ello, las velares y palatales son consonantes densas, y las dentales y labiales difusas. (1954: §42).

Combinando estas características se consigue de nuevo la caracterización binaria de rasgos distintivos:

Según esto, las pretendidas propiedades de localización se reducen a propiedades de resonancia, y en lugar de diversos órdenes opuestos multilateralmente, nos encontramos con dos rasgos diferenciales que oponen bilateralmente consonantes agudas a consonantes graves, y consonantes difusas a consonantes densas. (1954: §42). 
El resumen ( $\S \$ 49$ y 50) de este capítulo ("Función distintiva: los rasgos pertinentes") sufre una profunda remodelación en la segunda edición, hacia la solución y la notación de rasgos distintivos de Jakobson, Fant y Halle (1952). Será a partir de esta segunda edición cuando Alarcos incluya la descripción analítica de los rasgos distintivos del inglés, tomada de esta obra (1954: §50), a la vez que cree una descripción con los mismos principios de la lengua española, como veremos a continuación.

\subsection{Los rasgos pertinentes en la descripción del español}

La profundización en la influencia de Jakobson en la segunda edición se percibe también en la descripción de las oposiciones del español, basadas en los rasgos pertinentes ( $\S 107-8)$. Para desarrollar su propuesta de oposiciones, Alarcos parte de la lista de sonidos proporcionada por Navarro Tomás (1918). A partir del cotejo de pares mínimos, llega a su sistema de 19 fonemas consonánticos (§104). Después, procede a distinguir los rasgos pertinentes que oponen a los fonemas. Si bien el cuadro de oposiciones es el mismo a lo largo de las cuatro ediciones de la Fonología española, con una leve diferencia que veremos, en la exposición se presentan varias divergencias y una simplificación:

$1950: \$ 107$

Líquida / no líquida

Nasal / oral

Grave / aguda

Anterior / posterior

Oclusiva / fricativa

Lateral / vibrante

Sorda / sonora

Simple / múltiple
1954-1965:\$107

Líquida / no líquida

Nasal / oral

Grave / aguda

Densa / difusa

Interrupta / continua

Sorda / sonora

Tensa / floja

Los cambios de terminología obedecen al seguimiento más estrecho de la teoría binarista de Jakobson, tal como se sostiene ahora en los Preliminaries, que implica también una mayor simplificación en el número de oposiciones. Ya se ha comentado la sustitución de anterior / posterior por densa / difusa. También sustituye las dos oposiciones oclusiva / fricativa y lateral / vibrante por una oposición que las abarca a ambas: interrupta / continua. Escribe Alarcos que este par "opone las oclusivas a las fricativas, y, en las líquidas, las vibrantes a las laterales". Por último, reemplaza la terminología de Navarro Tomás de simple / múltiple por la jakobsoniana tensa / floja, para la que señala que aunque sea concomitante con la oposición sorda / sonora, es pertinente para diferenciar las vibrantes entre sí. A partir de la descripción binarista de los rasgos del inglés, de Jakobson, Fant y Halle, Alarcos (1954: §114) intenta la descripción del español ${ }^{13}$ :

\footnotetext{
Este cuadro sufrirá algunas modificaciones en la tercera edición, como veremos en la Figura 7.
} 


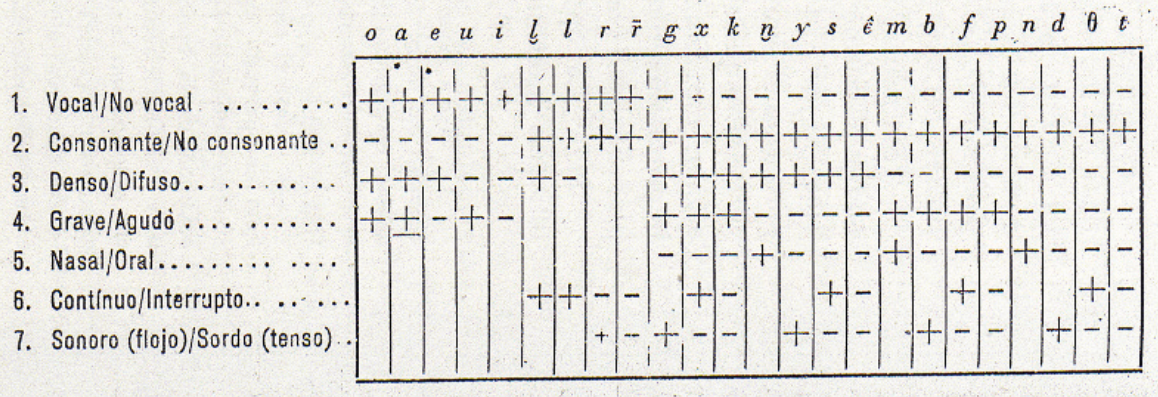

\section{MATIZACIÓN DEL BINARISMO}

Hay que hacer notar que el tono de logro que se registra en las dos primeras ediciones al referirse a Jakobson y el binarismo se difumina en gran medida en la tercera edición (1961), mostrándose Alarcos más moderado respecto del planteamiento binarista. Es preciso señalar que si en un principio podía parecer que el binarismo suponía una solución óptima en aras de la eficacia y la simplicidad de la descripción fonológica del sistema, pronto despertó suspicacias entre distintos sectores de la lingüística. Recogemos tan solo algunas de las críticas de autores que tuvo en cuenta Alarcos a partir de la tercera edición, que pueden explicar el cambio de actitud que se registra a partir de 1961.

\subsection{Críticas del binarismo}

Al otro lado del Atlántico, sin señalar a Jakobson, Hockett afirmó que "any phonemic system - and, indeed, any finite set of things whatsoever - can be arbitrarily converted to a binary code" (1955: 174). Sin embargo, tal operación es, desde el punto de vista fonológico, además de arbitraria, "a psychologic reductio ad absurdum", "un game-playing" (1955: 168). El lingüista debe evitar toda arbitrariedad en la descripción del sistema.

En Europa, la hipótesis binarista recibió una fuerte crítica por parte de Martinet, también muy influyente en Alarcos. Aunque se refiere a la cuestión en numerosas ocasiones a lo largo de su producción, nos fijamos en una obra que ciertamente influyó en Alarcos a partir de la tercera edición, la Economie des changements phonétiques, publicada en 1955 (Perea 2014). En esta obra, Martinet dedicó unas páginas al análisis del libro de Jakobson, Fant y Halle (1952). La cuestión se resumía como sigue: "Consideraciones teóricas han llevado a ciertos lingüistas a tratar de reducir todas las oposiciones fonológicas al tipo que parece prevalecer en el interior de los órdenes, es decir, al tipo binario" (1974 [1955]: 103).

Martinet señala que el binarismo se tendría que aceptar en dos supuestos: o bien, si se constata que todas las oposiciones fonológicas son binarias, mediante un análisis exhaustivo de las lenguas; o bien probar que el ser humano organiza sus unidades 
distintivas mediante un procedimiento dicotómico (1974 [1955]: 104). Ninguna de las dos posibilidades, señala, se han desarrollado lo suficiente.

Además, hay una serie de argumentos en contra. Desde el punto de vista articulatorio, no se puede defender el modelo binario, pues el modo de articulación de los sonidos tiene un carácter gradual (por ejemplo, en la abertura de las vocales). Aunque se demostrase, que también habría que hacerlo, que los niños adquieren el sistema fonológico por dicotomías sucesivas ${ }^{14}$, el hecho no implica que también así funcione el sistema del adulto. Martinet termina acusando a Jakobson de apriorismo teórico.

En cuanto al análisis en rasgos distintivos, se exige el realismo que impone la propia lengua:

El análisis de los fonemas en rasgos distintivos (en inglés distinctive features), tal y como lo practicamos en las páginas que siguen, no debe confundirse con el tipo de análisis que practican los binaristas. [...] mientras que nosotros hemos tendido siempre a eliminar todo lo que podía haber de subjetivo en el análisis trubetzkoyano y a no sacrificar jamás la originalidad de cada sistema, el binarismo, por el contrario, ha procedido a base de afirmaciones de carácter general y ha tratado de hacer entrar toda realidad fonológica en marcos preestablecidos. (Martinet 1974 [1955]: 96).

El lingüista sueco Bertil Malmberg, destacado por sus estudios en el campo de la Fonética, también se opuso al binarismo de Jakobson. Nos fijamos en un texto que también es recogido en la bibliografía de la Fonología de Alarcos, Lingüística estructural y comunicación humana. Escribe Malmberg:

La idea básica del concepto de rasgo distintivo, tal como es entendido por JakobsonFant-Halle, es que el receptor de un mensaje, cuando oye la onda sonora, se ve confrontado con una situación de elección doble y que en consecuencia tiene que elegir o bien entre dos cualidades polares de la misma categoría (grave - agudo, compacto difuso), o entre la presencia o ausencia de una determinada cualidad (sonora - sorda, nasalizada - no nasalizada). Así pues toda identificación de unidades fonológicas supone una elección binaria y el código fonológico es, por tanto, un código binario (1974 [1963]: 185)

Malmberg rechaza que la descodificación sea siempre de tipo binario y nunca una elección entre varias posibilidades. Propone el ejemplo del sistema vocálico del sueco, con tres grados de labialización. También recoge otras críticas a la teoría, debidas a Martinet y a Fry, este último desde el estudio sobre la percepción del lenguaje. En definitiva, Malmberg acusa la teoría de Jakobson de presentar "extrema simplificación y generalización" (1974: 193) ${ }^{15}$.

Martinet se refiere explícitamente a Jakobson (1941).

15 Por ejemplo, escribe "/i/ en el esquema dado más arriba puede ser identificado directamente como ni /u/ ni /a/, es decir, por su lugar en el sistema triádico" (1974: 193-4). 


\subsection{Las modificaciones de la tercera edición}

En primer lugar, nos fijamos de nuevo en el capítulo "Función distintiva: los rasgos pertinentes" (§§36-50), de la Fonología sincrónica. Encontramos un cambio de tono en la respuesta de la pregunta a la que hemos hecho referencia más arriba: “ ¿No habrá otras relaciones entre los fonemas de las diversas localizaciones, de manera que unos a otros se opongan bilateralmente?” (§41). La respuesta es distinta en las ediciones primera y segunda ("La solución de este problema parece haber sido alcanzado por Jakobson"), frente a la tercera y cuarta ("La solución dicotómica o binarista fue propuesta por Jakobson"). Asimismo, la conclusión de este capítulo se modifica con la supresión del adjetivo pretendidas, gracias a la cual se respeta el estatus de las propiedades de localización, que se negaba en las ediciones anteriores.

Según esto, las [1950-1954: pretendidas] propiedades de localización se reducen a propiedades de resonancia, y en lugar de diversos órdenes opuestos multilateralmente, nos encontramos con dos rasgos diferenciales que oponen bilateralmente consonantes agudas a consonantes graves, y consonantes difusas a consonantes densas. (1961: §42).

Finalmente, también se matizan las nuevas oposiciones al principio del epígrafe 45, en el mismo capítulo, en cuya redacción observamos la siguiente, significativa diferencia:

1950-1954

Las oposiciones grave / aguda y anterior / posterior [1954: difusa / densa] son bilaterales, equipolentes $[\ldots]$
1961-1965

En el caso de reconocer las oposiciones grave / aguda y difusa /densa, son éstas bilaterales, equipolentes $[\ldots]$

Quizá el mejor ejemplo de la vuelta a la oposición multilateral del lugar de articulación es un pasaje del epígrafe 44 donde se vuelve a la terminología articulatoria:

1950

En el sistema consonántico, una de las clases citadas (agudas anteriores, agudas posteriores, graves anteriores, graves posteriores) puede presentar divisiones*.
1961-1965

En el sistema consonántico, uno de los órdenes citados (labiales, dentales, prepalatales, dorsales) puede presentar divisiones.

En el capítulo "Los fonemas consonánticos" de la parte sobre Fonología del español, el desapego de la teoría dicotómica de la tercera edición se observará en los cambios de redacción de los epígrafes 107 y 108. En el primero se trata la descripción de los rasgos pertinentes que afectan al español. Como hemos visto, en la primera y segunda ediciones se refiere a las oposiciones dicotómicas, entre las que se sitúan el

\footnotetext{
Responden a la misma orientación las correcciones de la segunda edición: "En el sistema consonántico, uno de los órdenes citados (agudos difusos, agudos densos, graves difusos, graves densos) puede presentar divisiones".
} 
contraste grave / aguda y anterior / posterior (en la segunda edición, difusa / densa). En las ediciones tercera y cuarta, no presenta estas oposiciones directamente, sino que se refiere a ellas englobándolas en las diferencias de localización: "las diferencias de localización, con cuatro órdenes: labial, dental, alveolo-palatal y velar, que para los binaristas se reducen a la doble oposición densa / difusa [...], y grave/aguda [...]".

En la redacción del segundo epígrafe $(\S 108)$ también son perceptibles las diferencias:

$$
1950-1954
$$

En el sistema español, las oposiciones 3) y 4) se combinan, esto es, las oposiciones grave / aguda y densa / difusa se entrecruzan. El resultado es un sistema consonántico cuadrado...
$1961-1965$

Si se acepta el binarismo de las diferencias de localización señaladas en $\S 107,3)$, el resultado estructural es un sistema consonántico cuadrado...

Como se comprueba una vez más, Alarcos en las dos primeras ediciones presenta como suyo el enfoque que lleva a la descripción de las oposiciones del español, mientras que en las ediciones tercera y cuarta, lo relaciona con "los binaristas", la misma expresión que ha utilizado Martinet.

Asimismo, en el cuadro de oposiciones que aparece en el mismo epígrafe (§108), siendo el mismo, en la tercera y cuarta ediciones se restablece la información de localización. Véanse las figuras 2 y 3 :

Figura 2. Clasificación de fonemas (Alarcos 1950-1954: §108)

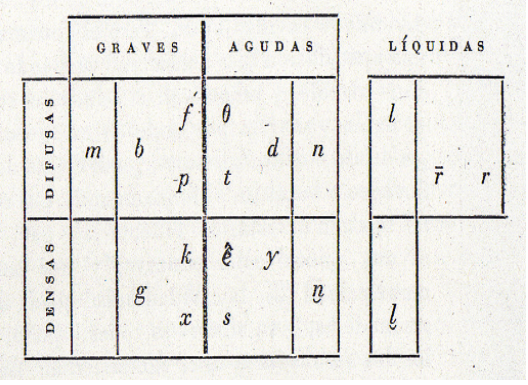

Figura 3. Clasificación de fonemas (Alarcos 1961-1965: §108)
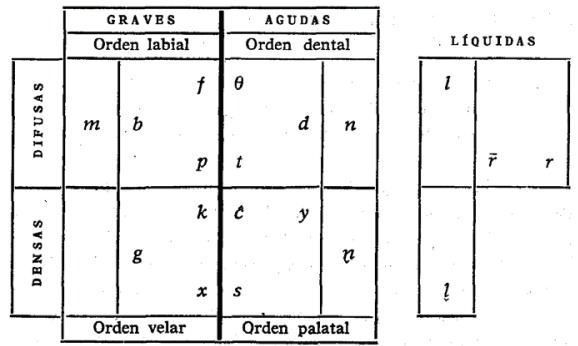

\section{UN NÚCLEO INALTERADO EN LA DESCRIPCIÓN DEL ESPAÑOL}

Como se ha analizado, el desarrollo de la fonología jakobsoniana ha condicionado modificaciones en la redacción de la Fonología española a lo largo de las tres primeras ediciones, primero como forma de acercamiento (entre la primera y la segunda edición) y luego como alejamiento (en la tercera). Sin embargo, comprobamos que hay una importantísima sección de la descripción del sistema consonántico del español que no sufre modificaciones en ninguna de las ediciones posteriores a la de 1950. 
En efecto, en los epígrafes 109-113, Alarcos desarrolla la clasificación de las oposiciones de las consonantes en correlaciones de sonoridad y continuidad que presuponen también las nociones de serie (entendida como grupo de fonemas que comparte un mismo modo de articulación) y orden (grupo de fonemas con el mismo punto de articulación) ${ }^{16}$. Se trata de conceptos propuestos por Trubetzkoy (1973: cap. 3) y que utiliza Martinet desde sus primeras publicaciones para clasificar las oposiciones frente a la metodología binarista. Alarcos incorpora estas categorías para establecer la clasificación de los fonemas consonantes según sus propiedades distintivas ${ }^{17}$.

Frente a los epígrafes 107-108, cuya evolución hemos analizado, se percibe ahora la necesidad de Alarcos de evitar la redundancia en la aplicación de los rasgos distintivos a la clasificación de los fonemas consonánticos. Esta necesidad recoge el imperativo de Trubetzkoy de no ofrecer rasgos redundantes en la definición fonológica ${ }^{18}$. También se intenta que en la definición fonológica se recojan todas las variantes del fonema, por ejemplo, cuando explica que "Los fonemas sonoros /b/, /d/, /g/ son indiferentes a la correlación plosiva - fricativa" ( $\$ 109)$, respetando así las variantes no oclusivas de estos fonemas ${ }^{19}$. Es evidente que en la descripción analítica que aparece en Jakobson, el concepto de rasgo pertinente deja de operar, ya que, si los fonemas han de caracterizarse en relación con un gran número de rasgos fonológicos, algunos de estos resultarán redundantes.

Desde una orientación martinetiana, la clasificación que ofrece Alarcos como resultado de las explicaciones anteriores se plasma en el epígrafe 114. Se trata de una clasificación en cinco series con cuatro órdenes implícitos, que rompen con los rasgos binarios que Jakobson había establecido para convertir la oposición multilateral del lugar de articulación en dos oposiciones bilaterales (y que, en cierta manera, contradicen el seguimiento que el propio Alarcos ha hecho de la obra de Jakobson). Hay que señalar que el diseño no sufre ninguna modificación a lo largo de las cuatro ediciones:

16 Martinet sistematiza la explicación como sigue: "Una lengua con $/ \mathrm{p} /, / \mathrm{t} /, / \mathrm{k} /, / \mathrm{m} /, / \mathrm{n} /, / \square /$ presentará dos series, una de nasales y otra de no nasales, y tres órdenes, labial, apical y dorsal. Series y órdenes presuponen una unidad mayor que los agrupa en un todo, la correlación, que comprende dos series paralelas y cierto número de fonemas pertenecientes a los mismos órdenes" (1974 [1955]: 99). Nótese que Alarcos ( $\$ 41-2)$ invierte la aplicación de los términos orden y serie entre la primera (en que sigue a Trubetzkoy 1973: 116) y las siguientes ediciones, dejando en las últimas el significado que les otorga el lingüista francés.

17 La explicación de las correlaciones por parte de Alarcos (1950-1965: §27) procede en última instancia de Trubetzkoy (1973: cap. 3). Pero junto a esta influencia fundamental, hay que tener en cuenta que en el procedimiento de análisis fonológico, desde la primera edición el catedrático de Oviedo tiene sigue de cerca también las publicaciones de Martinet (entre otras, 1936, 1945, 1947). De hecho, remite a ambos autores como referencia útil para la descripción fonológica, como aparece al final del epígrafe 21.

18 Escribe Trubetzkoy: "un fonema no contiene ningún rasgo fonológicamente no pertinente" (1973: 34; vid. en el mismo sentido 1973: 59). Se trata de una exigencia que acentúa Martinet (1940): "La segunda preocupación del lingüista es la de dar una definición estrictamente fonológica de las unidades funcionales que ha delimitado [... Se trata] de separar, entre las características articulatorias de este fonema, las que son pertinentes, esto es, aquellas sobre las que descansa esencialmente la función de distinguir este fonema de todos los demás fonemas del sistema (1968 [1940]: 59).

19 Hay que señalar, sin embargo, que este requisito no siempre se consigue, como ocurre cuando se definen como sordas $/ \theta / \mathrm{y} / \mathrm{s} /$. En tal definición dejan de recogerse los alófonos sonoros de estos fonemas en palabras como hazlo y mismo. 
Figura 4. Clasificación de fonemas (Alarcos 1950-1965: §114)

a) liquidas: $/ 1 /, / \mathrm{l} /, / \mathrm{r} /, / \overline{\mathrm{r}} /$

b) nasales: $/ \mathrm{m} /, / \mathrm{n} /, / \mathrm{n} /$

c) sonoras: $/ \mathrm{b} /, / \mathrm{d} /, / \mathrm{y} /, \mathrm{g} /$

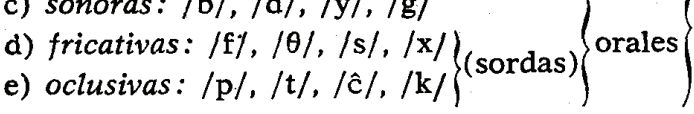

Nótese que esta clasificación recoge la forma de Martinet de marcar las oposiciones de los fonemas: las series en las líneas horizontales y los órdenes en las líneas verticales ${ }^{20}$. Si a este esquema se le incorporan los datos sobre el punto de articulación, obtenemos la clasificación de fonemas del español según los rasgos pertinentes, que sostiene Alarcos desde la primera a la última edición de su Fonología española:

Figura 5: Clasificación de fonemas de Alarcos a partir de $§ 114$ (Elaboración propia)

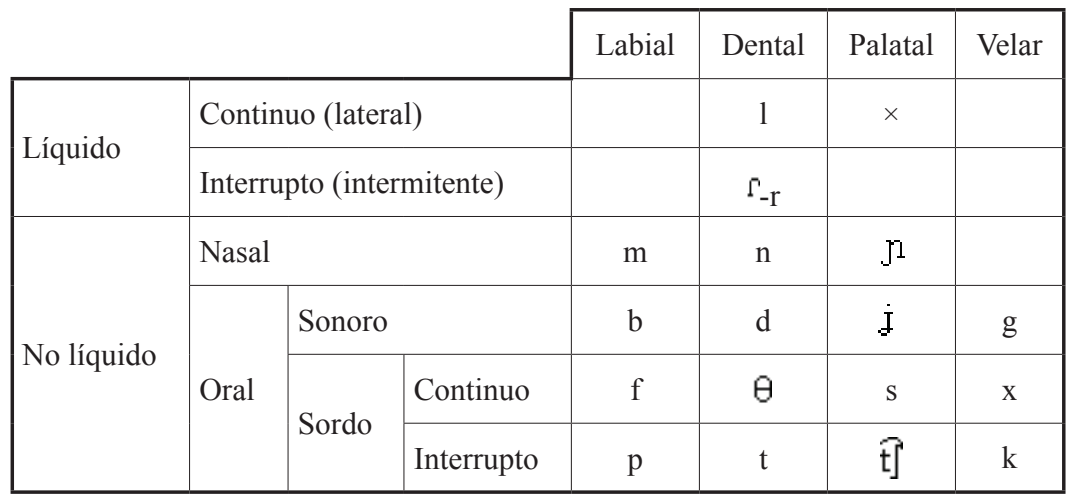

El cuadro resultante (Figura 5) se organiza atendiendo al lugar de articulación, siendo innecesarias las dos dicotomías jakobsonianas grave / agudo, denso / difuso. En horizontal se sitúan los rasgos relativos al modo de articulación, donde se incluye la acción de las cuerdas vocálicas.

Se trata, por otra parte, de la misma clasificación que aparecerá al final de la carrera de Alarcos, en la Gramática de la lengua española (1994), en la que se han simplificado los rasgos y recobrado la tradicional terminología articulatoria (Figura 6). Pero se trata del mismo cuadro:

20 De hecho, la misma organización para el español aparece en Martinet (1955: 3.26), con la diferencia de la interpretación fonológica de las semivocales. 
Figura 6: Clasificación de fonemas (Alarcos 1994: §17)

\begin{tabular}{|l|c|c|c|c|}
\cline { 2 - 5 } \multicolumn{1}{c|}{} & Labial & Dental & Palatal & velar \\
\hline Oclusiva (sorda) & $\mathrm{p}$ & $\mathrm{t}$ & $\mathrm{t}]$ & $\mathrm{k}$ \\
\hline Sonora & $\mathrm{b}$ & $\mathrm{d}$ & $\mathrm{j}$ & $\mathrm{g}$ \\
\hline Fricativa (sorda) & $\mathrm{f}$ & $\theta$ & $\mathrm{s}$ & $\mathrm{x}$ \\
\hline Nasal & $\mathrm{m}$ & $\mathrm{n}$ & $\mathrm{n}$ & \\
\hline $\begin{array}{c}\text { Líquidas } \\
\text { Vibrante } \\
\text { Lateral }\end{array}$ & & $\Gamma_{-r}$ & $\times$ & \\
\hline \multicolumn{2}{|c|}{} & & & \\
\hline
\end{tabular}

Para terminar, hay que hacer notar que inmediatamente después de la clasificación de fonemas consonánticos del epígrafe 114, se añade la definición de cada uno de los fonemas españoles en la que de nuevo aparecen todos los rasgos, lo que permite representar "los fonemas españoles, descompuestos en sus rasgos diferenciales", a imitación del proceder de Jakobson con la lengua inglesa ${ }^{21}$. Con este proceder, Alarcos describe los fonemas españoles según dos modelos alternativos: el de TrubetzkoyMartinet y el de Jakobson. No obstante, a partir de la tercera edición se incorporan cambios en este epígrafe: tanto las definiciones como el mismo cuadro analítico final se verán modificados en el proceso de depuración del binarismo que hemos descrito. En las definiciones, junto a los rasgos grave / agudo y denso / difuso, se recogerá ahora la localización, y, lo que es más significativo, en el cuadro analítico aparecerán entre paréntesis ciertos rasgos negativos, que resultan redundantes. Merece la pena reproducir el cuadro (Figura 7), que puede compararse con el que se muestra en nuestro epígrafe 3 (Figura 1):

Figura 7: Fonemas españoles (Alarcos 1961-1965: §119)

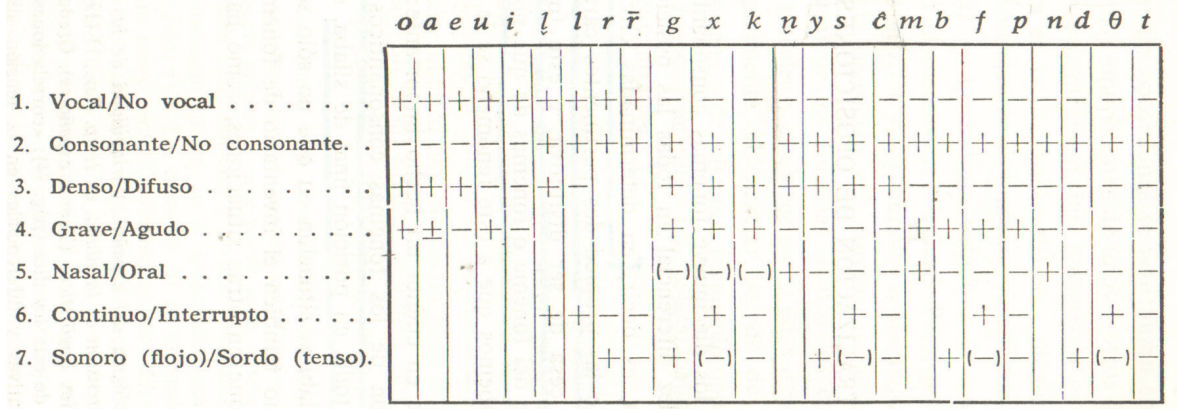

21 Así, por ejemplo, /f/ podía definirse según la clasificación que hemos visto simplemente como fonema labial, fricativo (de manera que se opone a los otros miembros de su correlación, /p/ y /b/ y al resto de correlaciones). En cambio, en el apartado de las definiciones aparece como "fonema no líquido, oral, difuso (frente a / $\mathrm{x} /$ ), grave (frente a $/ \theta /$ ), fricativo (frente a $/ \mathrm{p} /$ ), sordo (frente a $/ \mathrm{b} /$ ), como todas las fricativas" (1954: $\$ 114$ ), e igualmente aparece caracterizado por todos los "rasgos diferenciales" del sistema en el cuadro analítico del final de este epígrafe 114. 
Puede comprobarse que aparece entre paréntesis el rasgo nasal / oral en /g/, /x/, $/ \mathrm{k} /$, previsiblemente porque no hay fonemas velares nasales, y el rasgo sonoro / sordo en $/ \mathrm{x} /, / \mathrm{s} /, / \mathrm{f} /, / \theta /$, porque, según la exposición del autor, no hay fricativas sonoras a las que oponerse, ya que $/ \mathrm{j} /$ no ha sido definida como fricativa sino como no líquida, oral, palatal, sonora (probablemente, porque existe una variante africada).

\section{Conclusiones}

Hemos intentado dar cuenta en este artículo del influjo que la teoría binarista de Roman Jakobson ejerce en la Fonología española de Emilio Alarcos, teniendo en cuenta las modificaciones llevadas a cabo en el libro a lo largo de las cuatro ediciones de la obra. Como ha podido observarse, en el tratamiento de los fonemas consonánticos del español, Alarcos oscila entre una exposición que utiliza la metodología binarista de Jakobson y una orientación más deudora de la influencia de Trubetzkoy y Martinet. Por una parte, los epígrafes 107-108 son tributarios de la concepción teórica de Jakobson, en la forma de establecer los rasgos distintivos mediante oposiciones binarias. Hemos comprobado cómo en la segunda edición esta influencia se incrementa y cómo en la tercera disminuye, en lo que podríamos denominar un movimiento de ida y vuelta.

Por una parte, la exposición de los rasgos pertinentes del español (§107) se corresponde con las explicaciones de raigambre jakobsoniana de los epígrafes 49 y 50 del capítulo sobre "Fonología general", a la vez que desemboca en la descripción analítica de los rasgos distintivos del español, incorporada, como la del inglés, en la segunda edición (1954: $\S \S 50$ y 114). Por otra parte, los epígrafes 109-113, que estudian las correlaciones de la lengua española, están más influidos por la concepción de Trubetzkoy y Martinet, como hemos comprobado, en la búsqueda de una mayor simplicidad.

Por último, el epígrafe 114 recoge las dos tendencias que registra Alarcos en la descripción del consonantismo español. Si bien comienza con la clasificación de los fonemas en cinco series y cuatro órdenes, al modo de Trubetzkoy y Martinet, inalterada a lo largo de las cuatro ediciones, también aparecerán, deudoras de Jakobson, tanto las definiciones como la exposición analítica en rasgos diferenciales. Se trata de una muestra más del eclecticismo del lingüista salmantino, que no ha querido suprimir los puntos de vista que se defendían en ediciones anteriores (y ya no sostiene), sino que ha preferido limitarse a matizar ciertos extremos.

\section{AdDENDA: Alarcos en la obra de JaKobSON}

Durante los años que transcurren entre el artículo de Alarcos sobre el sistema fonológico del español (1949) hasta la tercera edición de la Fonología española (1961), Alarcos se sitúa como seguidor de la doctrina fonológica de Roman Jakobson, y se sitúa en la vanguardia de la fonología del español. El propio Jakobson se muestra conocedor de la obra de Alarcos, pues le cita en dos de sus producciones. La primera (Jakobson 1952), en un artículo en respuesta de una crítica de Robert A. Hall, Jr. a un libro escrito bajo las directrices del propio Jakobson. Se trataba de un 
enfrentamiento entre los dos tipos de estructuralismo, praguense y distribucionalista. Jakobson recuerda algunos autores que han seguido sus explicaciones en sus clases de Fonología de Nueva York (como P.L. Garvin, J. Mattoso Câmara e incluso Ch. F. Hockett). Y también señala que la misma línea de investigación fonológica han seguido otros autores, como Alarcos, de quien cita la primera edición de la Fonología española. Desde esta primera edición, Alarcos se había convertido en un referente necesario de la fonología española, al menos desde el enfoque del funcionalismo praguense. Cuatro años más tarde, Jakobson vuelve a citar a nuestro autor en el suplemento bibliográfico de su obra escrita con M. Halle (1956). En este caso, es la segunda edición de la monografía de Alarcos la que aparece.

A partir de estas referencias, se entiende que Jakobson conocía estas obras de Alarcos, no sabemos con qué detalle, en las que era reconocido como especialista y seguidor de las directrices de la fonología de Praga, concretamente en la orientación que siguió Jakobson tras la muerte de Trubetzkoy. Se entiende también que no aparezcan referencias a la obra de Alarcos a partir de la tercera edición, en la que el lingüista español se despega de los postulados binaristas más influido por las publicaciones de Martinet.

\section{OBRAS CITADAS}

Akamatsu, Tsutomu. 1988. The Theory of Neutralization and the Achiphoneme in Functional Phonology. Ámsterdam/Philadelphia: John Benjamins Publishing Company. Current Issues in Linguistic Theory.

Alarcos Llorach, Emilio. 1949. "El sistema fonológico español”. Revista de Filología Española 23: 265-296. . 1950 $1954_{2}, 1961_{3}, 1986$ [1965 $]$. Fonología Española. Madrid: Gredos. Biblioteca Románica Hispánica, 3. Manuales, 1.

. 1951. "Esbozo de una fonología diacrónica del español". Estudios dedicados a Menéndez Pidal. Vol. 2. Madrid: CSIC. 9-39.

. 1994. Gramática de la lengua española. Madrid: Espasa Calpe. Colección Nebrija y Bello.

Alcina, Juan \& José Manuel Blecua. 1989 [1975]. Gramática española. Barcelona: Ariel.

Anderson, Stephen R. 1990. La fonología en el siglo XX. Madrid: Visor.

Círculo Lingüístico de Praga. 1931. "Projet de terminologie phonologique standarisée". TCLP 4: 309-323.

Gil, Juana. 1989. “The binarity hypothesis in phonology (1938-1985)”, Historiographia Linguistica 16:1/2: 61-88.

Halle, Morris. 1954. "The Strategy of Phonemics", Word 15: 197-209.

1957. "In defense of number two", Studies Presented to Joshua Whatmough on his Sixtienth Birthday, ed. Ernst Pulgram. The Hague: Mouton. 65-72.

Hockett, Charles. 1955. A Manual of Phonology. Bloomington: Indiana University Press.

Jakobson, Roman. 1971 [1929]. "Remarques sur l'evolution phonologique du russe comparée a celle des autres langues slaves", en Jakobson, Roman. 1971. 1: 7-116. 1932. "Phoneme and Phonology", en Jakobson, Roman 1971. Vol. 1. 231-233. 1939. "Observations sur le classement phonologique des consonnes", Proceedings of the Third International Congress of Phonet Sciencies, Gante. Recogido en Jakobson, Roman. 1971. Vol. 1. 271-279. Trad. esp.: "Observaciones sobre la clasificación fonológica de las consonantes", Nuevos ensayos de lingüística general, de Roman Jakobson. Ma- 
drid: Siglo XXI, 1976. 131-139.

1941. Kindersprache, Aphasie und Allgemeine Lautgesetze. Upsala. Trad. esp. "Lenguaje infantil, afasia y leyes generales de la estructura fónica". Lenguaje infantil y afasia, de Roman Jakobson. Madrid: Ayuso, 1974. 17-137.

1949. "On the Identification of the Phonemic Entities", TCLP 5: 205-213. Trad. esp.

"La identificación de las entidades fonémicas", en Jakobson, Roman. 1984: 183-195.

1952. "For the Correct Presentation of Phonemic Problems", Word 8/4. Recogido en Jakobson, Roman. 1971. Vol. 1: 435-448.

1962. "Retrospect", en Jakobson, Roman. 1971. Vol. 1: 632-58. Trad. esp. "Panorama retrospectivo", en Jakobson, Roman. 1984: 139-182.

1971 [1962]. Selected Writings. The Hague: Mouton.

1984. Ensayos de lingüistica general. Barcelona: Ariel.

Jakobson, Roman, Gunnar Fant \& Morris Halle. 1952. Preliminaries to Speech Analysis. The Distinctive Features and Their Correlates. Cambridge Mass.: The MIT Press.

Jakobson, Roman \& Morris Halle. 1956. Fundamentals of Language. The Hague: Mouton. Trad. esp. de Carlos Piera: Fundamentos del lenguaje. Madrid: Ciencia Nueva, 1967.

Lepschy, Giulio C. 1971 [1966]. La lingüística estructural. Trad. esp. de Carlos Manzano. Barcelona: Anagrama.

Malmberg, Bertil. 1974 [1963]. Lingüiśstica estructural y comunicación humana. Madrid: Gredos.

Martinet, André. 1936. "Neutralisation et archiphoneme" TCLP 6: 46-57. 1940. "La phonologie syncronique et diachronique", Conférences de l'Institut de linguistique de l'Université de Paris, 6: 41-58. Revisado en Martinet, André. 1965: 50-65. 1945, "Description phonologique du parler franco-provençal d'Hauteville (Savoie)", Revue de linguistique romane 15: 1-86.

1947. “Où en est la phonologie?”, Lingua 1: 34-58. Revisado en Martinet, André, 1965: 65-82.

1955. Economie des changements phonétiques: Traité de phonologie diachronique. Berne: Editions A. Francke S.A. Trad. esp.: Economía de los cambios fonéticos: tratado de fonología diacrónica. Madrid: Gredos, 1974.

1957. "Substance phonique et traits distinctifs", Bulletin de la Société Linguistique 53: 72-85. Revisado en Martinet, André. 1965: 130-146.

1965. La linguistique synchronique: Études et Recherches. Paris: PUF. Trad. esp.: La lingüística sincrónica. Estudios e investigaciones. Madrid: Gredos, 1968.

Navarro Tomás. Tomás. 1972 [1918]. Manual de pronunciación española. Madrid: Publicaciones de la Revista de Filología Española, $17^{\mathrm{a}}$ ed.

Perea Siller, Francisco Javier. 2014. "Las cuatro ediciones de la Fonología española de Emilio Alarcos”, Métodos y resultados actuales en Historiografía de la Lingüística. Ed. María Luisa Calero, Alfonso Zamorano, Francisco Javier Perea, $\mathrm{M}^{\mathrm{a}}$ del Carmen García Manga \& María Martínez-Atienza. Vol. 2: 562-573. Münster: Nodus Publikationen.

Real Academia Española. 2011. Nueva gramática de la lengua española: Fonética y Fonología. Madrid: Espasa.

Trubetzkoy, Nikolai S. 1973[1939]. Principios de fonología. Trad. esp. Delia García Giordano con la colaboración de Luis J. Prieto. Madrid: Cincel. 
